\title{
Hàm sản xuất một mức với nhịp độ tăng năng suất tới hạn phụ thuộc mọi nhân tố sán xuất
}

\author{
Tô Cẩm Tú \\ Viẹn Quy Hoặch \& Thiết Ké Nông Nghiệp \\ Bọ Nóng Noghiẹp
}

\section{Mờ đầu}

Cho tới nay xuất hiện nhiêu phương pháp xây dụng hàm sản xuất (HSX), như dựa trên cơ sở các tiên dồ cồ điền về hàm sản xuất (xem $[2,3,4]$ ), hoặc sử dụng phương trình vi phân với độ co dãn thay thế không đồi (DCDTTKD) [3,5]. Nột số tác giả dà sứ dụng các hàm sản xuất với độ thay thế co giàn biến đồi (ĐCDTTBĐ) (xem $[1,6,7,8])$.

Gần đây với khái niệm nlị̣ độ tăng năng xuất tới hạn (NDTNSTH) được đưa vào cho hàm sản xuất cùng một số định lý cơ bạn (xem [9]) ta có tiêu chuân đề trên cơ sở bộ số liệu đề có thể xác định rằng hàm sản xuất tương ứng với nó có độ co giàn thay thế không đồi hay biến đồi. Biểu thức của nhịp độ tăng năng xuất tới hạn trong một nền sàn xuất nào đó còn cho biết hướng dầu tư (mờ rộng sàn xuất) đề đạt hiệu quả kinh tế cao. Trong [9] đà xây dựng các hàm sản xuât với độ co giãn thay thế không dồi và $\delta_{j i}=0, i, j=\overline{1 . n}, i \neq j$.

Trong bài này chúng tôi trình bày các hàm sản xuất với ĐCDTTKĐ mà $\delta_{j i}=$ $\delta_{j i}\left(x_{1}, x_{2}, \ldots, x_{n}\right)$ trong đó $x_{j}(j=\overline{1, n})$ là các nhân tố sản xuất.

\section{Dịnh nghĩa và Dịnh lý}

Hàm sản xuất một mức với độ co giàn thay thế không đồi và với nhịp hốn hợp tăng năng xuất giới hạn đa biến (phụ thuộc mọi nhân tố sản xuất) là hàm sản xuất: 


$$
y=f\left(x_{1}, x_{2}, \ldots, x_{n}\right)
$$

thỏa mãn:

$$
\begin{gathered}
\delta_{j j}+\frac{1}{\sigma x_{j}}=\delta_{j i}, \\
\delta_{j i}=\delta_{j i}\left(x_{1}, x_{2}, \ldots x_{n}\right)
\end{gathered}
$$

với mọi $i, j=\overline{1, n}, i \neq j$, trong đó:

$$
\begin{gathered}
\delta_{j j}=\frac{\frac{\partial^{2} y}{\partial x_{j}^{2}}}{\frac{\partial y}{\partial x_{j}}} \quad \text { là NDTNSTH dơn } \\
\delta_{j i}=\frac{\frac{\partial^{2} y}{\partial x_{j} \partial x_{i}}}{\frac{\partial y}{\partial x_{i}}} \quad \text { là NDTNSTH hỡn hợp }
\end{gathered}
$$

$\sigma=$ const. là độ co đãn thay thế các nhân tố.

Phương trình (1) trong định nghĩa chính là định lý cơ bản trong [9] cho hàm sàn xuất với độ co dãn thay thế không đồi còn $(2)$ chỉ ra sự phụ thuộc đa biến của $\delta_{j i}$.

Bồ đề: Trong mọi hàm sản xuất một mức với DCDTT không dồi NDTNSTH hồn hợp thóa mân hệ phuơng trinh sau:

$$
\frac{\partial \delta_{j i}}{\partial x_{i}}=\frac{\partial \delta_{i j}}{\partial x_{j}}
$$

vớ $\operatorname{mọ} i i, j=\overline{1, n}, i \neq j$.

Chứng minh. Đặt $p_{i}=\frac{\partial y}{\partial x_{s}}$ và cố định với mọi $x_{k}, k=\overline{1, n}, k \neq j$ thì

$$
\delta_{j i}=\frac{1}{p_{i}} \frac{d p_{i}}{d x_{j}}
$$

Lấy tích phân (4) theo $x_{j}$ ta được

$$
\int \delta_{j i} d x_{j}+\ln c_{j}=\int \frac{d p_{i}}{p_{i}}=\ln p_{i}
$$

hay:

$$
p_{i}=c_{j} \exp \int \delta_{j i} d x_{j}
$$

trong đó $c_{j}$ không phụ thuộc vào $x_{j}$.

Nếu $c_{j}$ phụ thuộc vào $x_{i}$ thì lấy đặo hàm (5) theo $x_{i}$ ta có:

$$
\frac{\partial^{2} y}{\partial x_{i}^{2}}=\frac{\partial p_{i}}{\partial x_{i}}=\left(\partial c_{j} / \partial x_{j}+c_{j} \int \frac{\partial \delta_{j i}}{\partial x_{j}} d x_{j}\right) e^{\int \delta_{j i} d x_{j}}
$$


Tì đó

$$
\delta_{i i}=\frac{\partial^{2} y}{\partial x_{i}^{2}} / \frac{\partial y}{\partial x_{i}}=\frac{1}{c_{j}} \frac{\partial c_{j}}{\partial x_{i}}+\int \frac{\partial \delta_{j i}}{\partial x_{i}} d x_{j}
$$

Do đó (1) trờ thành

$$
\frac{1}{c_{j}} \frac{\partial c_{j}}{\partial x_{i}}+\int \frac{\partial \delta_{j i}}{\partial x_{i}} d x_{j}+\frac{1}{\delta_{i j} x_{j}}=\delta_{i j}
$$

Lại lấy đạo hàm phương trình cuối cùng theo $x_{j}$ ta được phương trình (3).

Nếu $c_{j}$ không phài là hàm cuà $x_{i}$. thì $\frac{\partial c_{1}}{\partial x_{1}}=0$ và ta có ngay kết quà khi lấy đạo hàm phương trìuh cuối cùng theo $x_{j}$.

Ngược lại, lấy tích phân (3) theo $x_{i}$ ta được

$$
\delta_{j i}=\int \frac{\partial \delta_{i j}}{\partial x_{j}} d x_{i}+c_{i},
$$

trong đó $c_{i}$ không phụ thuộc vào $x_{i}$.

Rò ràng là

$$
\int \frac{\partial \delta_{i j}}{\partial x_{j}} d x_{i}=\delta_{j j}
$$

Tư đó suy ra rằng $c_{i}=c_{i}\left(x_{j}\right)$ và đặc biệt tự dịnh nghía rút ra $c_{i}=1 / \delta x_{j}$ dối với hàm sàn xuất với độ co làn thay thế không đồi. Bồ để dược chứng minh.

Chứng minh cách kháe:

$+a^{I}$ intr

Lấy đạo hàm (1) theo $x_{i}$ với chụ ỳ raằng d là hằng số ta dược

$$
\frac{\partial \delta_{j i}}{\partial x_{i}}=\frac{\partial}{\partial x_{i}}\left(\delta_{j j}+\frac{1}{\sigma x_{j}}\right)=\frac{\partial \delta_{j j}}{\partial x_{i}}=\frac{\partial^{3} y}{\partial x_{j}^{2} \partial x_{i}} / \frac{\partial y}{\partial x_{j}}-\delta_{j j} \delta_{i j} .
$$

Mặt khác theo dịnh nghỉa NDTNSTII hỡ hợp ta có

$$
\frac{\partial \delta_{i j}}{\partial x_{j}}=\frac{\partial}{\partial x_{j}}\left(\frac{\partial^{2} y}{\partial x_{i} \partial x_{j}} / \frac{\partial y_{-}}{\partial x_{j}}\right)=\frac{\partial^{3} y}{\partial x_{i} \partial x_{j}^{2}} / \frac{\partial y}{\partial x_{j}}-\delta_{i j} \delta_{j j}
$$

Tìr dó ta rút ra phương trìinh cần tìm.

Mệnh đề 1: Nhịp dộ hơn hợp tăng suất tới hạn cuả hàm sản xuất một mức $y=$ $f\left(x_{1}, x_{2}, \ldots x_{n}\right)$ vớ $i$ dọ co dìn thay thế không dồi thoả män

$$
\frac{\delta_{j i}}{\delta_{i j}}=a_{j i}\left(\frac{x_{i}}{x_{j}}\right)^{\frac{1}{0}},
$$

trong dó $\alpha_{j i}$ không phải là hàm. cuả $x_{i}$ và $x_{j}$ ló́i mọi $i, j=\overline{1, n} ; i \neq j$.

Chíng minh. Phương trình (6) là đương nnhiên, vì một mặt theo dịnh nghia của $\delta_{j i}$ và $\delta_{i j}$ ta có

$$
\frac{\delta_{j i}}{\delta_{i j}}=\left(\frac{\partial^{2} y}{\partial x_{j} \partial x_{i}}\right) /\left(\frac{\partial y}{\partial x_{j}} / \frac{\partial^{2} y}{\partial x_{j} \partial x_{i}} / \frac{\partial y}{\partial x_{j}}\right)=\frac{\partial y}{\partial x_{j}} / \frac{\partial y}{\partial x_{i}} .
$$


Mặt khác theo định nghià chuân thay thế

$$
\frac{\partial y}{\partial x_{j}} / \frac{\partial y}{\partial x_{i}}=-\frac{d x_{i}}{d x_{j}}
$$

và vì theo [9]

$$
\frac{d x_{i}}{d x_{j}}=-\alpha_{j i} \cdot\left(\frac{x_{i}}{x_{j}}\right)^{\frac{1}{\sigma}}
$$

trong đó $\alpha_{j i}$ là hằng số, ta có ngay (6).

Tuy nhiên ở đây ta chưng minh một các khác đề thấy rò $\alpha_{j i}$ là hằng số đối với những biến nào.

Tìr (5) ta có

$$
y=\int c_{j} e^{\int \delta_{y_{i}} d x_{j}} d x_{i}+K_{i}
$$

trong đó $c_{j}$ không phụ thuộc vào $x_{j}$ và $K_{i}$ không phụ thuộc vào $x_{i}$.

Hai lần lấy đạo hàm (5)' theo $x_{i}$ ta được

$$
\begin{gathered}
\frac{\partial y}{\partial x_{j}}=\int c_{j} e^{\int \delta_{j} d x_{j}} \delta_{j i} d x_{i}+\frac{\partial K_{i}}{\partial x_{j}} \\
\frac{\partial^{2} y}{\partial x_{j}^{2}}=\int c_{j} e^{\int \delta_{,} d x_{j}}\left(\delta_{j i}^{2}+\frac{\partial \delta_{j i}}{\partial x_{j}}\right) d x_{i}+\frac{\partial^{2} K_{i}}{\partial x_{j}^{2}} .
\end{gathered}
$$

Mặt khác, từ (1) suy ra

$$
\frac{\partial y}{\partial x_{j}}=\alpha_{j} x_{j}^{-\frac{1}{\sigma_{e}} \int \delta_{j,} d x_{j}}
$$

hay

$$
\alpha_{j}=\frac{\partial y}{\partial x_{j}} / x_{j}^{-\frac{1}{\sigma} \epsilon} \int \delta_{, j} d x_{j},
$$

trong đó $\alpha_{j}$ không phy thuộc vào $x_{j}$,

$$
\frac{\partial^{2} y}{\partial x_{j}^{2}}=\alpha_{j} x_{j}^{\frac{-1}{\sigma}} e^{\int \delta_{, j} d x_{j}}\left(\delta_{j i}-\frac{1}{\delta x_{j}}\right) .
$$

So sánh (7) với (9) và (8) với (11) ta được hệ phương trình

$$
\begin{gathered}
\int c_{j} e^{\int \delta_{j_{i}} d x_{j}} \delta_{j i} d x_{j}+\frac{\partial K_{i}}{\partial x_{j}}=\alpha_{j} x_{j}^{-\frac{1}{\sigma}} e^{\int \delta_{j i} d x_{j}}, \\
\int c_{j} e^{\int \delta_{j i} d x_{j}}\left(\delta_{j i}^{2}+\frac{\partial \delta_{j i}}{\partial x_{j}}\right) d x_{i}+\frac{\partial^{2} K_{i}}{\partial x_{j}^{2}}=\alpha_{j} x_{j}^{-\frac{1}{\sigma}} e^{\int \delta_{j i} d x_{j}}\left(\delta_{j i}-\frac{1}{\sigma x_{j}}\right)
\end{gathered}
$$


Lẩy đạo hàm liệ phương trình cuối cùng (chiu ý rằng $K_{i}$ không phụ thuộc vào $x_{i}$ ) theo $x_{i}$ ta được

$$
\begin{gathered}
c_{j} \delta_{j i}=x_{j}^{-\frac{1}{\sigma}}\left(\frac{\partial \alpha_{j}}{\partial x_{i}}+\alpha_{j} \int \frac{\partial \delta_{j i}}{\partial x_{i}} d x_{j}\right) \\
c_{j}\left(\delta_{j i}+\frac{\partial \delta_{j i}}{\partial x_{j}}=x_{j}^{-\frac{1}{\sigma}}\left[\left(\frac{\partial \alpha_{j}}{\partial x_{i}}+\alpha_{j} \int \frac{\partial \delta_{j i}}{\partial x_{i}} d x_{j}\right)\left(\delta_{j i}-\frac{1}{\sigma x_{j}}+\alpha_{j} \frac{\partial \delta_{j i}}{\partial x_{i}}\right] .\right.\right.
\end{gathered}
$$

Chia (13) cho (12), với chú ý rằng $\delta_{j i} \neq 0$ và do bô clề ta được

$$
\frac{1}{\delta_{j i}} \frac{\partial \kappa_{j i}}{\partial x_{j}}=\alpha_{j} \frac{\partial \delta_{j i}}{\partial x_{i}} /\left(\frac{\partial \alpha_{j}}{\partial x_{i}}+\alpha_{j} \delta_{j i}\right)-\frac{1}{\tau x_{j}}
$$

Ta chi̛ng minh rằng $\alpha_{j}=$ constant, tiic là không phụ thuộc vào bất cứ biến nào.

Thực vậy, già sừ ngược lại $a_{j}$ là hàm cria uhừng biốn khác với $x_{j}$. Khi dó tîr (10) ta dược

$$
\frac{\partial a_{j}}{\partial x_{i}}=x_{j}^{\frac{1}{\sigma}} e^{-\int \delta_{,} d x_{j}}\left[\frac{\partial^{2} y}{\partial x_{j} \partial x_{i}}-\frac{\partial y}{\partial x_{j}} \delta_{i j}\right] .
$$

Những biều thí̛c trong ngoặc bằng 0 (theo định nghĩa của nhịp độ hờn hợp tăng năng suất giới hậ), vì vậy $\frac{\partial \alpha_{1}}{\partial r_{1}}=0$ mâu thuân với già lhiết rằng $\alpha_{j}$ là hàm của $x_{i}$.

Do dó, theo bồ đề ta có

$$
a_{j} \frac{\partial \delta_{j i}}{\partial x_{i}} /\left(\frac{\partial a_{j}}{\partial x_{i}}+a_{j} \delta_{i j}\right)=\frac{1}{\delta_{i j}} \frac{\partial \delta_{i j}}{\partial x_{j}},
$$

và phương trình (11) bây giơs có d ạng

$$
\frac{1}{\delta_{j i}} \frac{\partial \delta_{j i}}{\partial x_{j}}-\frac{1}{\delta_{i j}} \frac{\partial \delta_{i j}}{\partial x_{j}}=-\frac{1}{\sigma x_{j}}
$$

Phương trình này cho nghiệm

$$
\frac{\delta_{j i}}{\delta_{i j}}=c_{j} x_{j}^{-\frac{1}{o}}
$$

trong đó $c_{j}$ không phụ thuộc $x_{j}$.

Tương tự, tì phương trinh

$$
\frac{1}{\delta_{i j}} \frac{\partial \delta_{i j}}{\partial x_{i}}-\frac{1}{\delta_{j i}} \frac{\partial \delta_{j i}}{\partial x_{i}}=-\frac{1}{\sigma x_{i}}
$$

ta dược

$$
\frac{\delta_{i j}}{\delta_{j i}}=c_{i} x_{i}^{-\frac{1}{a}},
$$

trong đó $c_{i}$ không phụ thuộc vào $x_{i}$. 
Toàn bộ chứng minh trên dẫn đến vấn để là $c_{j}$ là hàm của $x_{i}$ và $c_{i}$ là hàm của $x_{j}$, mà chính là $c_{j}=\beta_{j} x_{i}^{\frac{1}{\sigma}}, c_{i}=\beta_{i} x_{j}^{\frac{1}{\sigma}}$ trong đó $\beta_{j}$ và $\beta_{i}$ hoàn toàn không phụ thuộc vào $x_{i}$ và $x_{j}$. Thực vậy, giả sừ

$$
\frac{\delta_{j i}}{\delta_{i j}}=\frac{\beta_{j}}{\beta_{i}}\left(\frac{x_{i}}{x_{j}}\right)^{\frac{1}{\alpha}}
$$

Đưa đằng thức này về dạng lô - ga rít và lấy đạo hàm kết quả thu được theo $x_{j}$, ta được

$$
\frac{1}{\delta_{j i}} \frac{\partial \delta_{j i}}{\partial x_{j}}-\frac{1}{\delta_{i j}} \frac{\partial \delta_{i j}}{\partial x_{j}}=-\frac{1}{\sigma x_{j}}
$$

Từ đó hiêñ nhiên rằng hệ thức cần tỉm và (16) chỉ khác nhau một hằng số.

Mệnh đề 1 , về nguyên tăc, có thề được sử dụng để xây dựng mọi hàm sản xuất với độ co dãn thay thế không đồi, nhưng đó là một công cụ rất phức tạp. Vì vậy ta sẽ tiến hành bằng con đường khác (kém tổng quát hơn), và (5) khi đó có thêe được dùng đề kiêm tra tỉnh chỉnh xác của hàm sản xuất với độ co dãn thay thế không đồi đã được xây dựng.

Kí hiệu $\delta_{j j}^{y}, \delta_{j i}^{y}$ lần lượt là nhịp độ đơn và hổn hợp tăng năng suất tới hạn của hàm $y=f\left(x_{1}, \ldots x_{n}\right)$ và $\delta_{j j}^{Y}, \delta_{j i}^{Y}$ là các nhịp độ tương ứng của hàm $Y$, trong đó $Y$ là hàm số của $y$, ta có mệnh đề sau:

Mệnh dể 2. Hàm trơn cúa hàm sản xuất một mức với độ co dân thay thế không đồi là hàm với cùng độ co dãn thay thế không đời nó.

Chứng minh. Già sừ $y$ là hàm bất kỳ của $x_{1}, x_{2}, \ldots, x_{n}$ và $Y=F(y)$ là hàm liên tục và hai lần khá vi liên tục cuia $y$. Khi đó từ

$$
\begin{gathered}
\frac{\partial Y}{\partial x_{j}}=\frac{d Y}{d y} \frac{\partial y}{\partial x_{j}}, \frac{\partial^{2} Y}{\partial x_{j}^{2}}=\frac{d^{2} Y}{d y^{2}}\left(\frac{\partial y}{\partial x_{j}}\right)^{2}+\frac{d Y}{d y} \frac{\partial^{2} y}{\partial x_{j}^{2}}, \\
\frac{\partial^{2} Y}{\partial x_{j} \partial x_{i}}=\frac{d^{2} Y}{d y^{2}} \frac{\partial y}{\partial x_{j}} \frac{\partial y}{\partial x_{i}}+\frac{d Y}{d y} \frac{\partial^{2} y}{\partial x_{j} \partial x_{i}},
\end{gathered}
$$

ta dược

$$
\delta_{j j}^{Y}=\delta_{y y}^{Y} \frac{\partial y}{\partial x_{j}}+\delta_{j j}^{y}, \delta_{j i}^{Y}=\delta_{y y}^{Y} \frac{\partial y}{\partial x_{j}}+\delta_{j i}^{y}
$$

trong dó

$$
\delta_{y y}^{Y}=\frac{d^{2} Y}{d y^{2}} / \frac{d Y}{d y}
$$

Từ đó

$$
\delta_{j j}^{Y}-\delta_{j i}^{Y}=\delta_{j j}^{Y}-\delta_{i j}^{Y} .
$$

Vì vậy nếu $y$ là hàm sản xuất một mức với độ co dãn thay thế không đổi, tức là nếu

$$
\delta_{j j}^{y}+\frac{1}{\sigma x_{j}}-\delta_{j i}^{y}=0
$$


thi

$$
\delta_{j j}^{Y}+\frac{1}{\sigma x_{j}}-\delta_{j i}^{Y}=0
$$

theo định nghia $Y=F(y)$ cùng là hàm sản xuất một mức với độ co dàn thay thế không đồi $\sigma$. Dó là điểu phài chứng minh.

\section{Một vài ví dụ}

Sứ dụng định lý 2 mà các hàm cơ bản $y=f\left(x_{1}, \ldots x_{n}\right)$ là các hàm sản xuất co dãn thay thế không dồi và nhịp độ hỡn hợp tăng năng suất tới hạn bằng không (9).

$$
\begin{aligned}
& y=\sum_{j=1}^{n} \alpha_{j} \frac{\sigma}{\sigma-1} x_{j}^{\frac{\sigma-1}{\sigma}} \text { nếu } 1<\sigma<\infty
\end{aligned}
$$

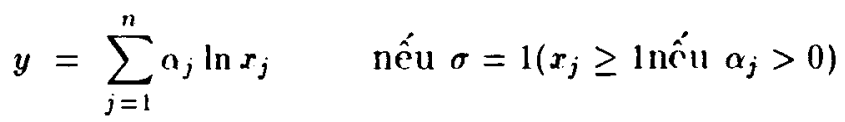

$$
\begin{aligned}
& y=\sum_{j=1}^{n} a_{j} x_{j} \quad \text { nếu } \sigma=\infty \\
& y=\min \left(x_{1}, x_{2}, \ldots x_{n}\right) \quad \text { nốu } \sigma=0
\end{aligned}
$$

ta có thể xây dựng hàng loạt hàm sàn xuất với dộ co dãn thay thế không đồi và nhịp dộ tăng năng suất tới hạn là hàm cuia các biến $x_{1}, x_{2}, \ldots x_{n}$.

Hàm sàn xuất $Y=a^{y}, a=$ constant. Dề dàng thấy rằng

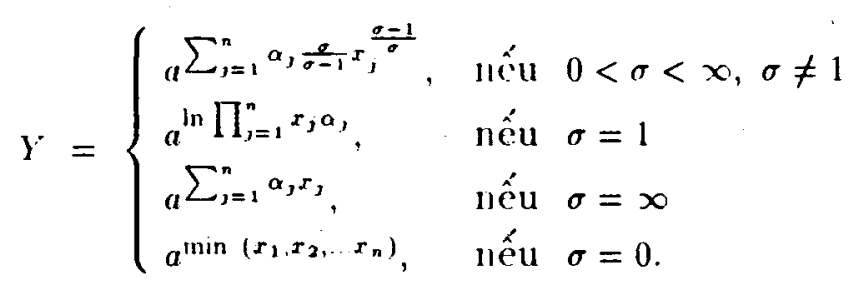

Trong dạng này ta có nhận xét sau

1. Nếu $a=\epsilon$ thì ta dược hàm Cobb-Douglas

$$
Y=\prod_{j=1}^{n} x_{j}^{\alpha},
$$

khi $\sigma=1$.

2. Khảc với trường hợp nhịp độ tăng hốn hợp năng suất tới hạn bằng không, trong hàm sản xuất dạng này $\delta_{j j}=f_{j}\left(x_{j}\right)$. Ilơn nữa, $\sigma$ có thể lấy mọi giá trị hằng số tưu 0 đến $\infty$.

3. Riêng với trường hợp $\delta=0$ hàm số vẫu có nhịp độ hốn hợp tăng năng suất tới hạn bằng không. 
Hàm sản xuất dạng $Y=b y^{a} ; a, b=$ constant.

1. Với $0<\delta<\infty, \delta \neq 1$, ta có

$$
Y=b\left[\sum_{j=1}^{n} \alpha_{j} \frac{\sigma}{\sigma-1} x_{j}^{\frac{\sigma-1}{\sigma}}\right]^{a}
$$

Với $a>0$ thì đây là hàm CES quen biết, và là hàm thuần nhất bậc $\frac{\sigma-1}{\sigma} a$.

- Nếu $a=\frac{\sigma}{\sigma-1}$ thì $Y$ tuyến tính thuân nhất.

- Hàm mờ rộng quy, mô sản xuất giàm nếu $a<\frac{\sigma}{\sigma-1}$ và tăng với $a>\frac{\sigma}{\sigma-1}$.

, Với $a<0$ thì $Y$ là hàm tỳ lệ nghịch với $y$.

Nhìn vào (17) ta thấy rằng $Y$ không âm với mọi giá trị của $a$, nếu $b>0$ và $\alpha_{j}>0$ nếu $\sigma>1, \alpha_{j}<0$ nếu $\sigma<1$. Đạo hàm bậc nhất không âm khi $a>0$ (nếu $\sigma>1$ ) và khi $a<0$ (nếu $\sigma<1$ ). Đạo hàm bậc hai $\partial^{2} Y / \partial x_{j}^{2}$ không dương khi

$$
\frac{\alpha_{j} x_{j}^{\frac{\sigma-1}{\sigma}}}{\sum_{j=1}^{n} \alpha_{j} x_{j}^{\frac{\sigma-1}{\sigma}}} \leq \frac{1}{(\sigma-1)(a-1)}
$$

Dặc biệt bất đằng thức cuối cùng luân luân thỏa mãn nểu $a \leq \frac{\sigma}{\sigma-1}$.

2. Với $\sigma=1$, ta có

$$
Y=b\left[\sum_{j=1}^{n} \alpha_{j} \ln x_{j}\right]^{a}
$$

Dễ dàng thấy rằng với cùng mức độ mờ rộng qui mô sản xuất thỉ hoàn trả (return) của nền sản xuất xác định bởi hàm (18) thấp hơn của nên kinh tế xác định bằng hàm (17). Hàm này thóa mãn mọi tiên đề của hàm sản xuất nếu $b>0, \alpha_{j}>0,0<a \leq 1$.

3. Với $\sigma=\infty$ thì hàm

$$
Y=b\left[\sum_{j=1}^{n} \alpha_{j} x_{j}\right]^{a}
$$

là hàm sản xuất nếu $b>0, \alpha_{j}>0,0<a \leq 1$.

Hàm sàn xuất dạng logarit $Y=\ln y$.

Nếu đặt

$$
Y=\ln \left[\sum_{j=1}^{n} \alpha_{j} \frac{\sigma}{\sigma-1}\left(x_{j}+a_{j}\right)^{\frac{\sigma-1}{\sigma}}\right]
$$

trong đó $a_{1}, \ldots a_{n}$ là những hàm sổ không âm bất kỳ, thỏa mãn

$$
\sum_{j=1}^{n} \alpha_{j} \frac{\sigma}{\sigma-1} x_{j}^{\frac{\sigma-1}{\sigma}}=1
$$

với $\alpha_{j} \geq 0, j=\widetilde{1, n}$ thì (19) là hàm sản xuất không thuần nhất.

Một cách tương tự ta xét cho các dạng mà $\sigma=1, \sigma=\infty$, và $\sigma=0$ (Léontiev). 
Hàm sản xuất dạng Logistic $Y=\frac{a}{1+c \exp (-1)}$.

Như đà biết, trong $[9,10]$ xem rằng sự tăng trương trong các nển kinh tế đang phát triền có thề nghiên cứu được nếu dùng hàm sản xuất lõm - lồi. Nếu xem năng suất cây trồng là hàm của lượng phân bón các loại được sử dụng cho một công thức canh tác nào đó thì ta luân có

$$
y_{0}=f(0,0, \ldots, 0)>0 \text {, }
$$

trong đó $y=f\left(x_{1}, x_{2}, \ldots, x_{n}\right)$ là năng xuất , và $x_{j}$ là lượng phân bón loại $j$. Hàm này trong một miền nhất định nào đó có thê xem như tuân theo luật logistic (lõm - lồi) v.v...

Đề nghiên cứu các dạng phát triên trên có thể dùng luật logistic.

Dề làm ví dụ chúng tôi xét hàm sản xuất với $0<\sigma<\infty, \sigma \neq 1$. Khi đó ta có

$$
Y=\frac{a}{1+\operatorname{cr} \overline{x p\left(-\sum_{j=1}^{n} \alpha_{j} \frac{\sigma}{\sigma-1} x_{j}^{\frac{\sigma-1}{\sigma}}\right.} .}
$$

Hàm (20) là hàm sản xuất nếu $a>0, f_{0}=a /(1+c), c>0$ (hoặc $-1<c<0$ ) hoặc với $a<0$ thì $-\infty<c<-1$ và $a_{j}>0$ nếu $a>0, c>0$ hoặc $a<0,-\infty<c<-1, \alpha_{j}<0$ nếu $a>0,-1<c<0$ với mọi $j=\overline{1, n}$.

Tóm lại, nhờ mệnh đề 2 ta có thề xây dụng hàng loạt hàm sản xuất với độ co dãn thay thế không đồi.

\section{Tài liệu tham kháo.}

1. Ivanilov U. P. \& Lotov A.B., Các mô hỉnh toán học trong kinh tê, M Nauka, 1979, 303 p. (in Russian).

2. Cobb C.W. \& Douglas P.H., A theory of production, Amer. Economic Rev. 1928, n18, p. 139-165.

3. Vladimir Strnad, Metody odvozeni jednotlirych typi produkenich funkci, Ekonomic - ko - matematicky obzor, Cesko-slovenska Akademie Ved , 1972, N1, p. 1-17.

4. Arrow K.J., Chenery II. B., Ninkas B. S. \& Solow R. N., Capital - Labour subsitution and economic efficientcy, Rev. Economics and Statistics, 1961, N 43, p. 225-250.

5. Yasui T., The CES production function, A note, Econometrica, July 1965, V. 33, p. 646-648.

6. Kleiner G. B., Hàm sản xuất M. Tài chính và Thống kê, 1986, 238 p. (in Russian).

7. Mikhalevskii $\mathrm{B}$. N., Hàm sản xuất và mô hỉnh tăng trưởng kinh tế, Kinh tế và các phương pháp toán học, 1967, t. III, N 2. p.199-222 (in Russian).

8. Allen R.G.D.; Macro-Economic Theory. A mathematical treatment, Macmillan, London, Melbourne, Toronto, St. Martin's Press, New-York., 1967, 420 p. 
9. Tô Cầm Tú. Về một số phương pháp xây dựng hàm sán xuất, Trung Tâm tính toán VHL Liên xô, M. 1988, 28 p. (in Russian).

10. To Cam Tu, The one-lcvel-production functions with the rates of limit productivity's growth of several variables, The $4^{\text {th }}$ Congress of Vietnamese Mathematicians, Hanoi September 4-7, 1990. p.133.

\section{Abstract}

The one level - production functions with the rates of limit productivity's growth of several variables.

By using of rates of limit productivity's growth

$$
\delta_{j i}=\frac{\partial^{2} y}{\partial x_{j} \partial x_{i}} / \frac{\partial y}{\partial x_{j}}, i, j=\overline{1, n}
$$

in this work, the following fundamental theorems for onc-level production functions with constant elasticity of substitution are proved:

a.

$$
\frac{\partial \delta_{j i}}{\partial x_{i}}=\frac{\partial \delta_{i j}}{\partial x_{j}}
$$

$b$.

$$
\frac{\delta_{j i}}{\delta_{i j}}=\alpha_{j i}\left(\frac{x_{i}}{x_{j}}\right)^{1 / \delta}
$$

With these sentences the building of all such functions is possible. However, for the construction of almost all such functions we have a simple theorem:

The smooth function of one-level production function with constant elasticity of substitution is the function with the same constant of elasticity of substitution. 